\author{
INSTITUT FÜR KERNPHYSIK, UNIVERSITÄT FRANKFURT \\ D - 60486 Frankfurt, August-Euler-Strasse 6, Germany
}

IKF-HENPG/1-98

\title{
On Transverse Momentum Event-by-Event Fluctuations in String Hadronic Models
}

\author{
Feng Liu円 \\ Institut für Kernphysik, Universität Frankfurt, Germany and \\ Institute of Particle Physics, Huazhong Normal University, Wuhan, China \\ An Tail2 \\ Institute of High Energy Physics, Beijing, China \\ Marek Gaździcki and Reinhard Stock \\ Institut für Kernphysik, Universität Frankfurt, Germany
}

\begin{abstract}
Transverse momentum event-by-event fluctuations are studied within the stringhadronic model of high energy nuclear collisions, LUCIAE. Data on non-statistical $p_{T}$ fluctuations in $\mathrm{p}+\mathrm{p}$ interactions are reproduced. Fluctuations of similar magnitude are predicted for nucleus-nucleus collisions, in contradiction to the preliminary NA49 results. The introduction of a string clustering mechanism (Firecracker Model) leads to a further, significant increase of $p_{T}$ fluctuations for nucleus-nucleus collisions. Secondary hadronic interactions, as implemented in LUCIAE, cause only a small reduction of $p_{T}$ fluctuations.
\end{abstract}

\footnotetext{
${ }^{1}$ E-mail: fliu@iopp.ccnu.edu.cn

${ }^{2} \mathrm{E}$-mail: taian@hptc1.ihep.ac.cn

${ }^{3} \mathrm{E}$-mail: marek@ikf.physik.uni-frankfurt.de

${ }^{4}$ E-mail: stock@ikf.physik.uni-frankfurt.de
} 


\section{Introduction}

Experimental results on event-by-event fluctuations in nuclear collisions at relativistic energy may serve as a crucial test of various theoretical approaches to the collision process. Until now the existing models were developed with the aim to reproduce the data on single particle yields and two particle correlations. To which extend can the same models predict event-by-event fluctuations?

A method to study fluctuations of global kinematical observables in high energy nuclear collisions (like fluctuations of total transverse momentum) was proposed in [1]. It was applied to analyze experimental data [2, 3] and test theoretical approaches [4, 5, 6]. The method is based on the fact that in elementary interactions (e.g. p+p interactions) particles are produced in a correlated way which leads to the observation of large (nonstatistical) event-by-event fluctuations. These 'elementary' fluctuations provide a scale relative to which the fluctuations in nuclear collisions can be studied in a model independent way. The method also provides a statistical tool to account for 'trivial' geometrical fluctuations.

Recently it was shown that initial state scattering models lead to increase of $p_{T}$ fluctuations [四]. This is in contradiction with the experimental results which indicate that the properly normalized $p_{T}$ fluctuations in central $\mathrm{Pb}+\mathrm{Pb}$ collisions at $158 \mathrm{~A} \cdot \mathrm{GeV}$ are significantly smaller than the corresponding fluctuations in $\mathrm{p}+\mathrm{p}$ interactions [2, 7]. To which level can the experimental result be understood as an effect of equilibration due to hadronic cascading? What is the role of possible collective effects at the early stage of the collision, like clustering of strings? The aim of this paper is to investigate these questions using the string-hadronic model LUCIAE [8]

The paper is organized as follows. The analysis method used further to study eventby-fluctuations is recalled in Section 2. In the Section 3 we briefly sketch the basic physics ingredients of the LUCIAE. Model calculations concerning $p_{T}$ fluctuations are presented and discussed in Section 4. Summary and discussion close the paper.

\section{A Measure of Event-by-Event Fluctuations}

Event-by-event fluctuations in nuclear collisions are usually dominated by the trivial variation in impact parameter from event to event and by the purely statistical (here we mean statistics for classical particles) variation of the measured quantities. An analysis method that allows to remove these trivial contributions and to determine the remaining part of event-by-event fluctuations of transverse momentum has been proposed in [1]. Following this reference we define for every particle $i$ in an event:

$$
z_{i}=p_{T_{i}}-\overline{p_{T}}
$$

where $\overline{p_{T}}$ is the mean transverse momentum of accepted particles averaged over all events (the inclusive mean). Using $z_{i}$ we calculate for every event

$$
Z=\sum_{i=1}^{N} z_{i}
$$


where $N$ is the number of accepted particles in the event. With this definition one obtains the following measure of event-by-event fluctuations:

$$
\Phi_{p_{T}}=\sqrt{\frac{\left\langle Z^{2}\right\rangle}{\langle N\rangle}}-\sqrt{\overline{z^{2}}},
$$

where $\langle N\rangle$ and $\left\langle Z^{2}\right\rangle$ are averages over all events and the second term in the r.h.s. is the square root of the second moment of the inclusive transverse momentum distribution. The physical motivation for studying $\Phi_{p_{T}}$ was given in [1] : experimental data on $\mathrm{N}+\mathrm{N}$ interactions show that particles in these collisions are not produced independently [9]. One observes large scale correlations that lead to, e.g., a correlation between the event multiplicity and the average $p_{T}$ of the particles. The correlated particle emission in elementary processes can be used to probe the dynamics of nuclear collisions by measuring to which degree this correlation is changed when going to $\mathrm{p}+\mathrm{A}$ and/or $\mathrm{A}+\mathrm{A}$ collisions.

For this purpose, $\Phi_{p_{T}}$ as a measure of fluctuations has two important properties. For a large system (i.e. an $\mathrm{A}+\mathrm{A}$ collision) that is a superposition of many independent elementary systems (i.e. $\mathrm{N}+\mathrm{N}$ interactions), $\Phi_{p_{T}}$ has a constant value that is identical to that of the elementary system. In other words if the central $\mathrm{Pb}+\mathrm{Pb}$ collisions were an independent superposition of $\mathrm{N}+\mathrm{N}$ interactions, the value of $\Phi_{p_{T}}$ would remain constant, independent of the number of superimposed elementary interactions in a single event and its distribution in the studied sample of the events. If on the other hand the large system consists of particles that have been emitted independently, $\Phi_{p_{T}}$ assumes a value of zero. Thus $\Phi_{p_{T}}$ provides us with a scale characterising the fluctuations in nuclear collisions relative to elementary interactions at the same energy.

One should expect that $\Phi_{p_{T}}$ is sensitive to both event-by-event fluctuations in the creation of the early state of the collision as well as in its subsequent evolution until freeze-out.

\section{The string-hadronic model - LUCIAE}

The LUCIAE model is developed based on FRITIOF [10]. In the FRITIOF model a hadron is assumed to behave like a massless relativistic string corresponding to a confined color force field of a vortex line character embedded in a type II color superconducting vacuum. In FRITIOF, during the collision two hadrons are excited due to longitudinal momentum transfers and/or Rutherford Parton Scattering (RPS). The highly excited states will emit bremsstrahlung gluons according to the soft radiation model. They are afterwards treated as excitations, i.e. the Lund Strings, and allowed to decay into final state hadrons according to the Lund fragmentation scheme [11].

The LUCIAE includes all elements of the FRITIOF model and, additionaly, two components: a 'Firecracker' model and a model of hadron rescattering. In the Firecracker model it is assumed that groups of neighbouring strings may form interacting quantum states (clusters) so that both the emission of gluonic bremsstrahlung as well as the fragmentation properties can be affected by the large common energy density of the interacting strings. The maximum transverse momentum of the emitted gluons is found to fulfill a condition [12] $k_{\perp \max } \leq \sqrt{\mu M_{\text {tot }}}$, where $M_{\text {tot }}$ is the total excitation energy of a cluster and 
$\mu M_{\text {tot }}$ effectively corresponds to an energy density over a region of transverse size $1 / \mu$. Consequently, when it comes to heavy ion collision predictions the Firecracker model will correspond to an essential enhancement of (mini)jets in the center of phase space, which contributes to high $p_{T}$ enhancement [12].

In the rescattering model, the produced particles (which consist of hadrons after strong decays and the participant nucleons) are distributed randomly in the geometrical overlapping region between the projectile and the target nuclei. The target (projectile) spectator nucleons are distributed randomly outside the overlapping region and inside the target (projectile) sphere. A formation time is given to each particle and a particle starts to scatter with others after it is "formed". Two particles will collide if their minimum distance $d_{\text {min }} \leq \sqrt{\sigma_{\text {tot }} / \pi}$, where $\sigma_{\text {tot }}$ is the total cross section in $\mathrm{fm}^{2}$ and the minimum distance is calculated in the cms frame of the two colliding particles (for details see [8]). 


\section{The $p_{T}$ Fluctuations in LUCIAE}

The analysis of the event-by-event fluctuations in LUCIAE is done in several steps. In the first part of this section we study event-by-event fluctuations in $\mathrm{p}+\mathrm{p}$ interactions. In the following subsections the $p_{T}$ fluctuations in $\mathrm{A}+\mathrm{A}$ collisions are analyzed. We study effects of independent string superposition, string clustering and finally the role of the hadronic rescattering.

In the analysis presented in this paper the $\phi_{p_{T}}$ variable was calculated using all charged particles stable with respect to strong interaction, charged spectator fragments were excluded. The number of generated events for all analyzed event samples was larger than $10^{4}$. The statistical error of the $\phi_{p_{T}}$ variable was calculated according to formula given in the Appendix.

\subsection{Fluctuations in $\mathrm{p}+\mathrm{p}$ Interactions}

It is well established experimenatlly [9] that particle production in $\mathrm{p}+\mathrm{p}$ interactions at high energy is correlated. It was pointed out in Ref. [1] that this correlation could lead to a large non-statistical event-by-event fluctuations which can be quantified by the $\Phi$ measurement.

It is essential that the string-hadronic models which are used for the analysis of the event-by-event fluctuations in $\mathrm{A}+\mathrm{A}$ collisions are first checked as to whether they reproduce the corresponding fluctuations measured in $\mathrm{p}+\mathrm{p}$ interactions. This is because in these models the latter process is used as an input for the calculation of the properties of the $\mathrm{A}+\mathrm{A}$ collisions.

It was shown [1] that the $\Phi_{P_{T}}$ variable is sensitive to the correlation between the form of the $p_{T}$ distribution and the event multiplicity. Therefore in Fig. 1 we compare the experimental data on $\left\langle p_{T}\right\rangle$ vs $n$ dependence for $\mathrm{p}+\mathrm{p}$ interactions at $200 \mathrm{GeV}$ [13] with the LUCIAE results. The comparison is done separately for $\pi^{+}$and $\pi^{-}$mesons. Two versions of LUCIAE are used. In Figs. 1a and 1c the results for the full version of the model, which includes hard processes (RPS), are shown. The results obtained by switching off hard processes in the model are presented in Figs. 1b and 1d. We note here that the string clustering model as well as hadronic rescattering play no role for $\mathrm{p}+\mathrm{p}$ interactions.

The comparison indicates that LUCIAE underpredicts both the absolute magnitude and the strength of the $\left\langle p_{T}\right\rangle$ vs $n$ dependence. The hard scattering processes, which occur in about $5 \%$ of $\mathrm{p}+\mathrm{p}$ interactions at $200 \mathrm{GeV}$, play a minor role when the $\left\langle p_{T}\right\rangle$ vs $n$ dependence is considered.

The fact that the $\left\langle p_{T}\right\rangle$ vs $n$ dependence is underpredicted by LUCIAE may suggest that the event-by-event $p_{T}$ fluctuations are smaller in the model than in the data. This is however not the case. The $\Phi_{p_{T}}$ value calculated within LUCIAE without hard processes is about $15 \mathrm{MeV} / \mathrm{c}$ for $\mathrm{p}+\mathrm{p}$ interaction at $158 \mathrm{GeV}$. This value of $\Phi_{p_{T}}$ agrees with the corresponding preliminary experimental value obtained by the NA49 Collaboration [7] when the acceptance effects are taken into account. The introduction of hard scattering increases the $\Phi_{P_{T}}$ value to $30 \mathrm{MeV} / \mathrm{c}$ leading to an overestimation of the experimental number.

The above results illustrate two important features of $\Phi_{p_{T}}$. Its value is determined not 
only by the $\left\langle p_{T}\right\rangle$ vs $n$ dependence but also by other correlations present in the particle production process (e.g. jet production). It is sensitive to different properties of the production process than the inclusive or semi-inclusive observables and therefore yields additional information otherwise not available.

The strong increase of the $\Phi_{p_{T}}$ value when the hard processes are included may be understood as due to the fact that the final state particles originating from the hard process (jets) are strongly correlated in momentum space.

In order to trace the origin of the particle correlation in the $\mathrm{p}+\mathrm{p}$ interactions for soft processes in LUCIAE we exchanged the string fragmentation scheme by an independent fragmentation scheme in which energy-momentum and quantum charges are not conserved. This results in a reduction of the $\Phi_{p_{T}}$ value by a factor of about 5 , to almost zero. Thus we conclude that the conservation laws are responsible for large event-by-event fluctuations in soft $\mathrm{p}+\mathrm{p}$ interactions at SPS energies in LUCIAE.

For the further study of $p_{T}$ fluctuations in $\mathrm{A}+\mathrm{A}$ collisions we selected the LUCIAE version without hard processes as only this version reproduces correctly the magnitude of the $p_{T}$ fluctuations measured for $\mathrm{p}+\mathrm{p}$ interactions [7].

\subsection{Superposition of Strings}

The fundamental assumption of the string-hadronic models of $\mathrm{A}+\mathrm{A}$ collisions is that the basic physics of these collisions can be pictured as an (almost) independent superposition of nucleon-nucleon $(\mathrm{N}+\mathrm{N})$ interactions.

Thus in order to study properties of this minimal model with respect to $p_{T}$ fluctuations we analyze the fluctuations calculated within the LUCIAE without hard processes, string clustering and hadronic rescattering. For this version of the model we calculate the $\Phi_{p_{T}}$ for central $(b=0 \mathrm{fm}) \mathrm{A}+\mathrm{A}$ collisions and plot it as a function of $A$ in Fig. 2.

One observes a independence of $\Phi_{p_{T}}$ of $A$. This behaviour is expected, by definition, for a model in which $\mathrm{A}+\mathrm{A}$ is assumed to be an independent superposition of $\mathrm{N}+\mathrm{N}$ interactions. In the studied version of LUCIAE there are two effects which can cause deviations from the independent superposition picture. The nucleons in the nucleus have Fermi motion and the string excitation increases with the size of the colliding nuclei. Our numerical results show that both effects play a minor role when the $\Phi_{p_{T}}$ is studied. Therefore, in this respect, the minimal version of the string-hadronic model of $\mathrm{A}+\mathrm{A}$ collisions can be considered as an independent superposition of $\mathrm{N}+\mathrm{N}$ interactions.

\subsection{String Clustering}

In the high density stage of the $\mathrm{A}+\mathrm{A}$ collisions the picture of independent string superposition is obviously unrealistic. A modification of this picture is modeled, in the framework of string-hadronic models, by introduction of string clustering (Firecracker model) [8] or string fusion [14].

In the Firecracker model clustering of strings allows for a collective gluon emission and therefore is expected to increase the $p_{T}$ fluctuations. The probability that the string will form a cluster increases with the size of the colliding nuclei and consequently the $p_{T}$ fluctuations due to string clustering should increase with the size of the colliding nuclei. In 
fact this is observed in Fig. 2, where the results of the calculations with string clustering effect are shown. For central $\mathrm{Pb}+\mathrm{Pb}$ collisions the value of $\Phi_{p_{T}}$ increases by a factor of about 3 when the string clustering is added to the minimal version of the LUCIAE model.

\subsection{Rescattering}

The early stage of $\mathrm{A}+\mathrm{A}$ collisions is followed by the stage in which hadrons and hadronic resonances are effective degrees of freedom. Considerable rescattering between them is expected to take place before the final decoupling of produced particles. For the interpretation of the measured event-by-event fluctuations it is crucial to understand the role played by the hadronic rescattering process.

The $\Phi_{p_{T}}$ calculated for central $\mathrm{S}+\mathrm{S}$ and $\mathrm{Pb}+\mathrm{Pb}$ collisions within the minimal version of the LUCIAE supplemented by the hadronic rescattering model is shown in Fig. 2. One observes that the $p_{T}$ fluctuations are slightly reduced by the hadronic secondary interactions. This trend can be, in fact, understood as the rescattering should lead to the equilibration of the final state and therefore it should reduce the fluctuations established at the early stage. From that point of view it may even be surprising that the role of the rescattering is relatively small, as each produced hadron rescatters in average 6 timesi until decoupling in central $\mathrm{Pb}+\mathrm{Pb}$ collision modeled by the present version of the LUCIAE. 


\section{Summary and Discussion}

The analysis of event-by-event fluctuations in string-hadronic model LUCIAE presented in this paper was triggered by the experimental results of the NA49 Collaboration [2, 4]. They indicate that $p_{T}$ fluctuations, as measured by $\Phi_{p_{T}}$ measure, are significantly reduced in central $\mathrm{Pb}+\mathrm{Pb}$ collisions at $158 \mathrm{~A} \cdot \mathrm{GeV}$ in comparison to $\mathrm{p}+\mathrm{p}$ interactions at the same energy.

Our main results can be summarized as follows.

1. The $\Phi_{p_{T}}$ value in $\mathrm{p}+\mathrm{p}$ interactions modeled by LUCIAE is greater than zero. This is caused by correlations between particles introduced by conservation laws and by hard scattering.

2. The $\Phi_{p_{T}}$ value is independent of the size of the colliding nuclei when $\mathrm{A}+\mathrm{A}$ collisions are modeled by the minimal version of the LUCIAE model (without hard scattering, string clustering and hadronic rescattering). It remains constant at the value found for $\mathrm{p}+\mathrm{p}$ interactions.

3. A string clustering effect, as introduced in LUCIAE, causes a strong increase of $\Phi_{p_{T}}$ with increasing size of the colliding nuclei.

4. Final state hadronic rescattering, as modeled in LUCIAE, only weakly decreases the value of $\Phi_{p_{T}}$.

Our results should be confronted with the recently presented results on event-by-event fluctuations as measured by the $\Phi_{p_{T}}$.

It was found in 4 that initial state scattering models predict an increase of $\Phi_{p_{T}}$ with the size of the colliding nuclei. This behaviour is similar to the behaviour obtained by us for the string clustering effect. The relative contribution of both effects (initial state scattering and string clustering) increases with the size of the colliding nuclei.

The influence of hadronic rescattering on event-by-event fluctuations was studied in Ref. [3] using the VENUS model [16] and in Ref. [5] using the UrQMD model [17]. The secondary interactions as modelled by VENUS were shown not to influence fluctuations in pseudorapidity measured by $\Phi_{\eta}$ [3]. UrQMD finds a strong reduction of the value of $\Phi_{p_{T}}$ when going from $\mathrm{p}+\mathrm{p}$ interactions to central $\mathrm{Pb}+\mathrm{Pb}$ collisions. This result is in contradiction with our finding (see point 4 above). We are aware of the following differences between the current analysis and the analysis done within UrQMD model [5, 18]. In the UrQMD analysis all particles at midrapidity $\left(-0.5<y^{*}<0.5\right)$ are used whereas in our analysis only charged particles but without rapidity selection are included. We checked however that our conclusion on the weak influcence of rescattering remains unchanged when a 'UrQMD acceptance' is used for LUCIAE events. The number of central $\mathrm{Pb}+\mathrm{Pb}$ events with rescattering was 10000 for LUCIAE and 1600 for UrQMDP. The rescattering prescriptions are different in UrQMD and LUCIAE. We checked however that the number of secondary hadronic collisions in central $\mathrm{Pb}+\mathrm{Pb}$ collision is similar (about 6 rescatterings per final state hadron).

\footnotetext{
${ }^{5}$ In both models generation of a single $\mathrm{Pb}+\mathrm{Pb}$ event takes about 30' CPU time on Pentium Pro 200.
} 
The difference in the conclusions may be, also, due to the fact that in UrQMD model Pauli blocking of baryons is taken into account. This leads us to a comment on the results obtained recently by Mrówczyński [6]. He calculated the value of $\Phi_{p_{T}}$ for an equilibrium ideal gas in the grand canonical approximation. In the case of classical particles $\Phi_{p_{T}}=$ 0 . The $\Phi_{p_{T}}$ value for fermions is large negative, whereas for bosons large positive. He estimates that due to the dominance of pions in the final state the value of $\Phi_{p_{T}}$ in the hadronic gas should be large and positive and therefore it is not easy to understand the NA49 results even assuming full equilibration of the matter.

We conclude from the above sketchy review that the basic discrepancy between the results of various models remains to be reconciled. Vis a vis the preliminary data obtained by NA49 [2, 7] which indicate a vanishing $\Phi_{p_{T}}$ measure both the results of our microscopic string-hadronic model LUCIAE, and expectation based on an equilibrium hadron quantum gas miss the mark. On the other hand the UrQMD model which is based on a similar physical picture predicts a vanishing $\Phi_{p_{T}}$ measure. Further, ongoing work is devoted to pinpoint the origin(s) of this apparent discrepancy. We also urgently await final affirmation of the preliminary NA49 data.

\section{Acknowledgements}

We would like to thank H. Ströbele and G. Roland for discussion and comments. This work was partly supported by DFG (Germany) and National Natural Science Fundation (China). 


\section{Appendix}

A. The fluctuation measure $\Phi_{x}$, where $x$ is any single particle variable, defined by Eq. 1 can be expressed in an explicit way by single event variables $N, X$ and $X_{2}$, where $\mathrm{N}$ is the number of particles and

$$
X=\sum_{i=1}^{N} x_{i}, \quad X_{2}=\sum_{i=1}^{N} x_{i}^{2}
$$

It is easy to show that the definition (Eq. 1) is equivalent to

$$
\Phi_{x}=\left(\frac{\left\langle X^{2}\right\rangle}{\langle N\rangle}-\frac{2\langle X\rangle\langle X N\rangle}{\langle N\rangle^{2}}+\frac{\left\langle N^{2}\right\rangle\langle X\rangle^{2}}{\langle N\rangle^{3}}\right)^{1 / 2}-\left(\frac{\left\langle X_{2}\right\rangle}{\langle N\rangle}-\frac{\langle X\rangle^{2}}{\langle N\rangle^{2}}\right)^{1 / 2} .
$$

The above formula allows to calculate value of $\Phi_{x}$ during a single pass of data processing, without initial evaluation of the inclusive mean, $\bar{x}$.

It is common to study event-by-event fluctuations by the analysis of the variance of the ratio $X / N$ (the mean $x$ for particles from a given event). From the definition of variance we get:

$$
V\left(\frac{X}{N}\right)=\left\langle\frac{X^{2}}{N^{2}}\right\rangle-\left\langle\frac{X}{N}\right\rangle^{2} .
$$

We make a trivial observation that the $\Phi_{x}$ and $V(X / N)$ are different functions of different moments of basic single event observables $X$ and $N$. Thus analysis of $\Phi_{x}$ is not equivalent to the analysis of $V(X / N)$.

B. One can estimate a statistical error of the $\Phi_{x}, \sigma\left(\Phi_{x}\right)$, when the single event variables $N, Z^{2}$ and $Z_{2}\left(Z_{2}=\sum_{i=1}^{N} z_{i}^{2}\right)$ are considered as the original random variables. The $\Phi_{x}$ can be then written as:

$$
\Phi_{x}=\left(\frac{\sum_{j=1}^{N_{E V}} Z_{j}^{2}}{\sum_{j=1}^{N_{E V}} N_{j}}\right)^{1 / 2}-\left(\frac{\sum_{j=1}^{N_{E V}} Z_{2, j}}{\sum_{j=1}^{N_{E V}} N_{j}}\right)^{1 / 2},
$$

where $N_{E V}$ is the number of events. The $\sigma\left(\Phi_{x}\right)$ can be therefore expressed as:

$$
\begin{aligned}
\frac{\sigma^{2}\left(\Phi_{x}\right)}{N_{E V}}= & \left(\frac{\delta \Phi_{x}}{\delta Z^{2}}\right)^{2} V\left(Z^{2}, Z^{2}\right)+\left(\frac{\delta \Phi_{x}}{\delta Z_{2}}\right)^{2} V\left(Z_{2}, Z_{2}\right)+\left(\frac{\delta \Phi_{x}}{\delta N}\right)^{2} V(N, N)+ \\
& \frac{\delta \Phi_{x}}{\delta Z^{2}} \frac{\delta \Phi_{x}}{\delta N} V\left(Z^{2}, N\right)+\frac{\delta \Phi_{x}}{\delta Z_{2}} \frac{\delta \Phi_{x}}{\delta Z^{2}} V\left(Z_{2}, Z^{2}\right)+\frac{\delta \Phi_{x}}{\delta N} \frac{\delta \Phi_{x}}{\delta Z_{2}} V\left(N, Z_{2}\right),
\end{aligned}
$$

where $\delta \Phi_{x} / \delta\left(N, Z^{2}, Z_{2}\right)$ is a derivate of $\Phi_{x}$ over a single event variable $\left(N, Z^{2}, Z_{2}\right)$ and $V\left(Y_{1}, Y_{2}\right)=\left\langle\left(Y_{1}-\left\langle Y_{1}\right\rangle\right)\left(Y_{2}-\left\langle Y_{2}\right\rangle\right)\right\rangle$. Of course finally the expression for the statistical error of $\Phi_{x}$ can be written in terms of the algebraic moments as in the case of the observable $\Phi_{x}$ itself (Eq. 2). 


\section{References}

[1] M. Gaździcki, St. Mrówczyński, Z. Phys. C54 (1992) 127.

[2] G. Roland (NA49 Collab.), Proceedings of the Hirschegg Workshop on QCD Phase Transitions, 1997 page 309.

[3] M. L. Cherry et al. (KLM Collab.), Acta Phys. Pol. B29 (1998) 2129.

[4] M. Gaździcki, A. Leonidov and G. Roland, On Event-by-Event Fluctuations in Nuclear Collisions, hep-ph/9711422 (1997), to be published in Eur. Phys. J. C.

[5] M. Bleicher et al., Can Momentum Correlations Proof Kinetic Equilibration in Heavy Ion Collisions at 160 A GeV, hep-ph/9803345 (1998), to be published in Phys. Lett. B.

[6] St. Mrówczyński, Transverse momentum and energy correlations in the equilibrium system from high-energy nuclear collisions, nucl-th/9806089, to be published in Phys. Lett. B.

[7] C. Adler et al. (NA49 Collab.), GSI Scientific Report, GSI 98-1 (1998).

[8] Sa Ben-Hao and Tai An, Computer Physics Commun., 90 (1995) 121.

[9] A. I. Golokhvastov, Z. Phys. C26 (1984) 469.

[10] H. Pi, Computer Physics Commun. 71(1992) 173.

[11] B. Andersson, G. Gustafson, G. Ingelman and T. Sjöstrand, Phys. Rep. 97, 31 (1983).

[12] B. Andersson, Phys. Lett., B256 (1991) 337; B. Andersson and Tai An, Z. Phys., C71 (1996) 155.

[13] T. Kafka et al. , Phys. Rev. D16 (1977) 1261

[14] M. A. Braun and C. Pajares Nucl. Phys. B390 (1993) 542,

H. Sorge, M. Berenguer, H. Stöcker and W. Greiner, Phys. Lett. B289 (1992) 6.

[15] G. Gustafson, Lund University preprint LU TP 92-26, Budapest Heavy Ion (1992) 1.

[16] K. Werner, Phys. Rep. 232 (1995) 87.

[17] S. A. Bass et al., Part. Nucl. 41 (1998) 225.

[18] M. Bleicher private communication. 

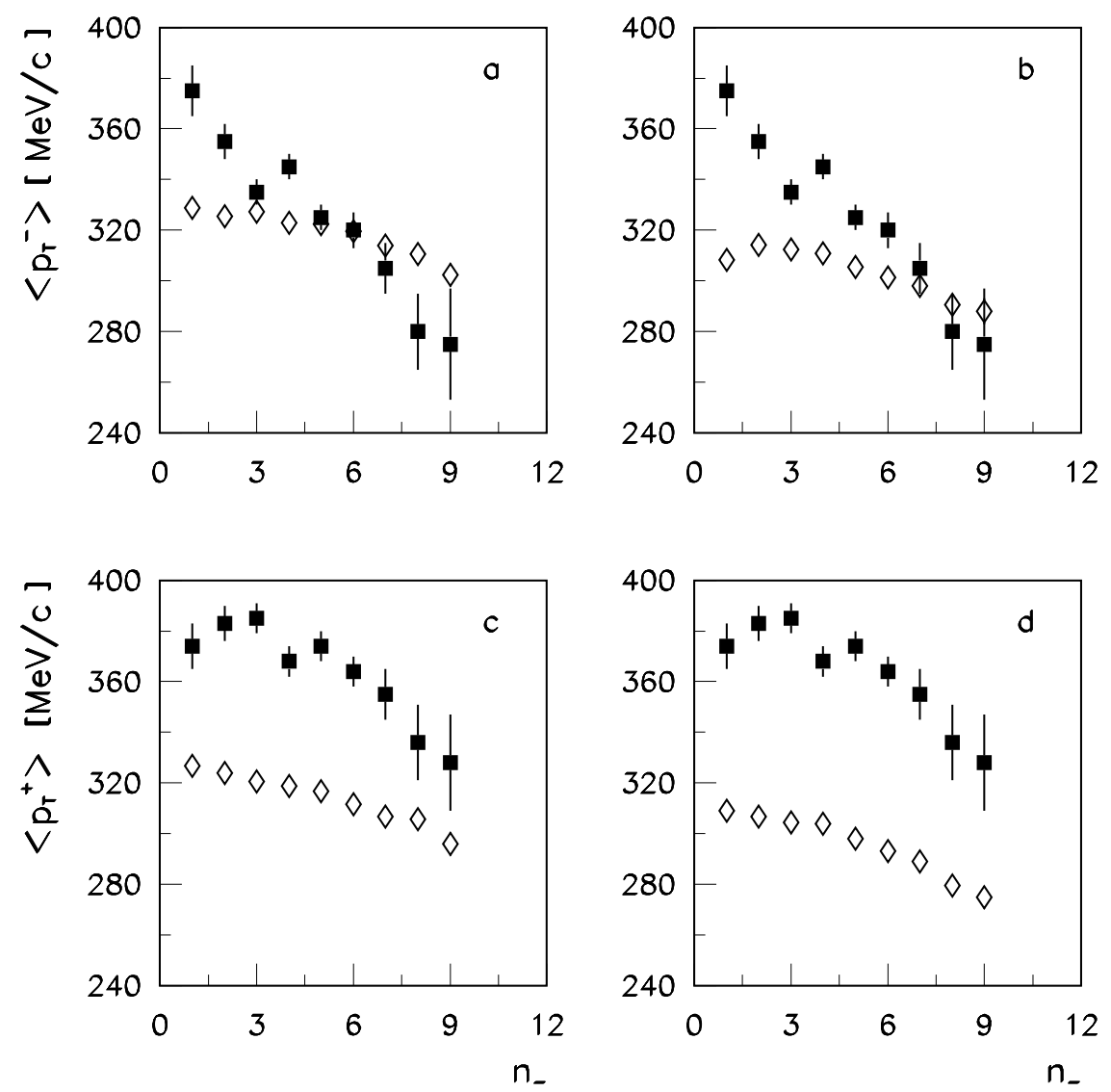

Figure 1: The dependence of the average transverse momentum of $\pi^{-}$(Figs. 1a and 1b) and $\pi^{+}$(Figs. 1c and 1d) mesons on multiplicity of negatively charged hadrons for $\mathrm{p}+\mathrm{p}$ interactions at $200 \mathrm{GeV}$. The experimental results [13] are indicated by the filled squares, whereas the results obtained within the LUCIAE model by the open diamonds. The hard processes are included in the case of Figs. 1a and 1c, and excluded in the case of Figs. $1 \mathrm{~b}$ and $1 \mathrm{~d}$. 


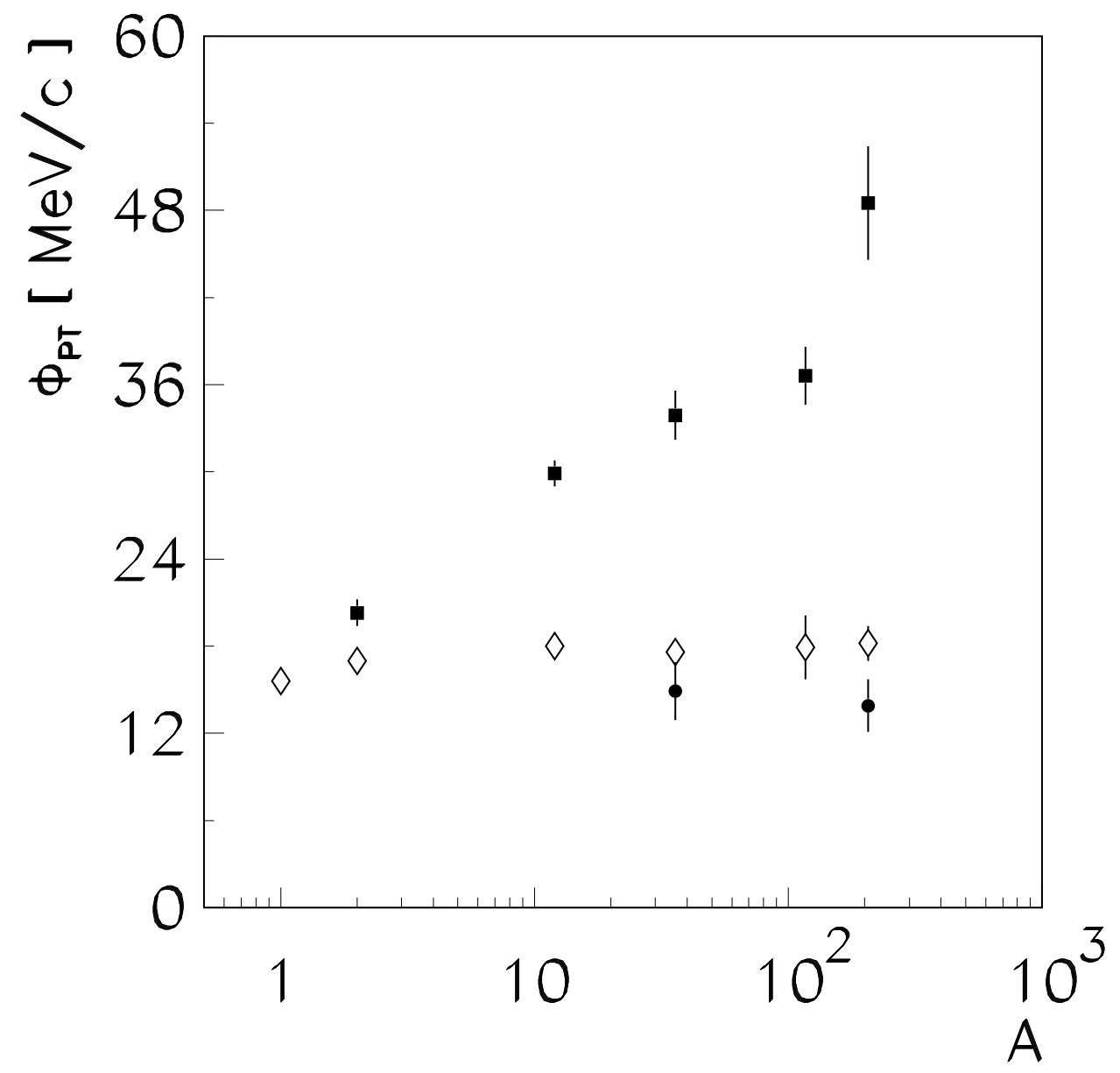

Figure 2: The dependence of the $\Phi_{p_{T}}$ on the nuclear mass number of the colliding nuclei calculated for $\mathrm{A}+\mathrm{A}$ collisions at $158 \mathrm{~A} \mathrm{GeV}$ at zero impact parameter using LUCIAE model. Results obtained without hard scattering, string clustering and secondary hadronic interactions (minimal version of the model) are indicated by open diamonds. Behaviour obtained by including string clustering is shown by filled squares. Filled circles indicate the value of $\Phi_{p_{T}}$ obtained when the hadronic rescattering is added to the minimal version of the model. 